hospital for an average of 30.9 days. I would suggest that they, and other long-stay patients, have post-mortems done by the hospital pathologists, thus leaving more time $\frac{\mathbb{\Phi}}{\Omega}$ for the coroner's pathologists to do more acute cases, including trauma. Ideally however, I would like to see multiple trauma post-mortems performed in the hospital $\stackrel{\infty}{\circ}$ by a pathologist with a specific interest in the subject but perhaps that is too revolutionary an idea, especially for London!

M. HOCKING

Accident and Emergency Consultant

Lewisham Hospital

London, England

\title{
Carpometacarpal dislocation: an unusual complex injury of the hand
}

\section{Sir}

We would like to report an uncommon case of carpometacarpal dislocation in a 30-year old man following a fall from a motorcycle at speed.

On arrival in hospital he had multiple superficial abrasions and marked swelling and $\vec{\bullet}$ tenderness over the right first and second metacarpals.

Radiographs of the right hand were interpreted as showing a spiral fracture of the fourth metacarpal shaft. This diagnosis was confirmed subsequently in the radiologis report. No other abnormality was noted.

He was immobilized in a plaster of Paris slab and on review of this 2 weeks later the $\frac{\partial}{\not}$ swelling was much reduced, but it was noted that the thumb appeared abnormal and there was an obvious bony lump at the base of the second metacarpal which appeared $\stackrel{\circ}{\exists}$ shortened. Further radiographs with oblique views (Figs. 1 and 2) showed that the first and second carpometacarpal joints were dislocated. Tomography confirmed the diagnosis. He was transferred to the Orthopaedic Department and underwent open $\mathbb{D}$ reduction and internal fixation of the joint.

Subluxation or dislocation of a carpometacarpal joint other than of the thumb is $\frac{5}{3}$ uncommon (Ho et al., 1987) because of strong ligamentous and bony stabilizers, and the $\delta$ diagnosis may easily be overlooked. A report of carpometacarpal dislocations by Henderson \& Arafa in 1987 showed 15 of 21 patients reviewed had the diagnosis missed 음 in the accident and emergency department and furthermore the injury was missed again 7 in five cases when they were reviewed by the senior registrar or consultant in orthopaedic surgery.

Hazlett (1968) suggests that there are two main mechanisms of injury. The common- N est is a blow to the metacarpophlangeal joint, the head of the metacarpals or the dorsum $\mathrm{N}_{\mathrm{N}}^{N}$ of the hand, probably with the wrist in palmar flexion. The alternative mechanism is palmar pressure exerted by motorcycle handlebars, as in this case.

Radiological diagnosis is difficult because routine radiographs may not show the displacement clearly. It has been suggested that a true lateral radiograph (Henderson $\&_{0}$ 
Arafa, 1987) is of most value but in our case (Fig. 1) the dislocation of the second carpometacarpal joint is not recognizable on this view.

Treatment by closed reduction is often successful when the injury is recognized immediately. Delay, as in this case, may mean that the dislocations need open reduction and internal fixation.

The prognosis when the injury is recognized and treated is excellent, even as late as 2 months. Untreated the dislocations disrupt both the longitudinal and transverse arches of the hand resulting in an impaired grip and loss of normal axial length. In conclusion, this is an uncommon injury that is easily missed, especially when it occurs in combination with a common, obvious injury. Dislocation of the carpometacarpal joints should be considered when generalized swelling of the dorsum of the hand is present after an injury, even with no obvious abnormality on standard radiographs.

This case also serves as a reminder that the discovery of one abnormality on a radiograph should lead to a search for another.

\section{F. GIBSON \& M. J. CLANCY}

Accident and Emergency Department

St. James Hospital

Leeds, England

\section{REFERENCES}

Hazlett J. W. (1968). Carpometacarpal dislocations other than the thumb: a report of 11 cases. The Canadian fournal of Surgery, 11, 315-22.

Henderson J. J. \& Arafa M. A. M. (1987). Carpometacarpal dislocation. The fournal of Bone and foint Surgery 69-B. 2.

Ho P. K. et al. (1987). Complex dorsal dislocation of the second carpometacarpal joint. fournal of Hand Surgery 12, 1074-6. 